\title{
Cladóceros fitófilos (Crustacea, Branchiopoda) do Parque Nacional das Emas, estado de Goiás
}

\author{
Francisco Diogo Rocha Sousa ${ }^{1}$; Lourdes Maria Abdu Elmoor-Loureiro ${ }^{1,2}$
}

${ }^{1}$ Laboratório de Zoologia, Universidade Católica de Brasília - UCB, QS 7 lote 1, Bloco M sala 331, CEP 72030-170, Taguatinga, DF, Brasil,

${ }^{2}$ Autor para correspondência: Lourdes Maria Abdu Elmoor-Loureiro, e-mail: lourdes@ucb.br, http://www.ucb.br

Sousa, F. D. R.; Elmoor-Loureiro, L. M. A. Phytopilous cladocerans (Crustacea, Branchiopoda) of the Parque Nacional das Emas, State of Goiás. Biota Neotrop., vol. 8, no. 1, Jan./Mar. 2008. Available from: <http://www. biotaneotropica.org.br/v8n1/en/abstract?inventory+bn02008012008>.

Abstract: This paper presents a preliminary inventory of the phytophylous cladocerans from the Parque Nacional das Emas. Eleven qualitative samples were taken from different sites (lagoon, vereda, and wet campo), from 2000 to 2006 . Eighteen species, belonging to typically non-planktonic families, were identified; the most specious family was the Chydoridae. All species are recorded for the first time in Southwest region of Goiás.

Keywords: phytofilous fauna, Anomopoda, cerrado, biodiversity, inventory.

Sousa, F. D. R.; Elmoor-Loureiro, L. M. A. Cladóceros fitófilos (Crustacea, Branchiopoda) do Parque Nacional das Emas, estado de Goiás. Biota Neotrop., vol. 8, no. 1, jan./mar. 2008. Disponível em: <http://www. biotaneotropica.org.br/v8n1/pt/abstract?inventory+bn02008012008>.

Resumo: Este trabalho apresenta um inventário preliminar de cladóceros fitófilos do Parque Nacional das Emas. Onze amostras qualitativas foram coletados de diferentes ambientes (lagoa, vereda e campo úmido), no período de 2000 a 2006. Foram identificadas 18 espécies, pertencentes a famílias tipicamente não planctônicas, sendo Chydoridae a mais especiosa. Todas elas representam o primeiro registro para a região sudoeste de Goiás.

Palavras-chave: fauna fitófila, Anomopoda, cerrado, biodiversidade, inventário. 


\section{Introdução}

As zonas litorais de ecossistemas aquáticos continentais são consideradas áreas de transição entre o ambiente terrestre e aquático, recebendo influência dos dois sistemas. Nesses ecótonos ocorrem microalgas, briófitas, pteridófitas e macrófitas aquáticas, que lhes conferem características ecológicas únicas.

A entrada de vários tipos de sedimentos oriundos dos sistemas terrestres, aliada a um banco de macrófitas diversificado, proporciona à zona litoral uma grande heterogeneidade de microhabitats, favorecendo o estabelecimento de organismos diversos (Raizer \& Amaral 2001, Nogueira et al. 2003), como fases imaturas de insetos, moluscos, anelídeos e crustáceos. Contudo, o conhecimento sobre a biota e o funcionamento dessas áreas ainda é reduzido, principalmente considerando os organismos que vivem associados à vegetação das zonas litorais, também chamados de organismos fitófilos.

Segundo Elmoor-Loureiro (2000), desde o século XIX estudos limnológicos e taxonômicos envolvendo cladóceros vêm sendo desenvolvidos no Brasil, embora que bastante concentrados na Região Sudeste e focados na comunidade zooplanctônica. Até o momento, poucos trabalhos foram realizados sobre os cladóceros do estado de Goiás. Lansac-Tôha et al. (1999) avaliaram a comunidade zooplanctônica do reservatório de Corumbá, constatando a ocorrência de 16 espécies de cladóceros, sendo nove delas pertencentes a famílias não planctônicas. Um inventário dos cladóceros fitófilos do vale do Rio Paranã é apresentado por Elmoor-Loureiro (2007), no qual são reportadas 39 espécies. Nenhuma referência se tem para a região sudoeste de Goiás, onde está localizado o Parque Nacional das Emas.

No Cerrado, o estudo de áreas alagadas também é bastante limitado, com poucos trabalhos publicados (Reid 1984, 1987, 1993 e 1994). Estas áreas apresentam uma grande diversidade de microorganismos (Reid 1993), além de ampla distribuição no Cerrado e algumas características exclusivas, como solo altamente orgânico, água levemente ácida, bem oxigenada e pobre em íons (Padovesi-Fonseca 2005).

Este trabalho tem com objetivo avaliar a composição da fauna fitófila de cladóceros do Parque Nacional das Emas, a partir de amostras qualitativas coletadas entre 2000 e 2006.

\section{Material e Métodos}

\section{1. Área de estudo}

O Parque Nacional das Emas possui aproximadamente 133.000 ha e está situado na região central do Brasil, mais precisamente no sudoeste do estado de Goiás, fazendo limite com os estados de Mato Grosso e Mato Grosso do Sul. (Figura 1)

O bioma predominante é o Cerrado, subdividido em várias fitofisionomias, como mata ciliar, vereda, vegetação ripária, campos úmidos.

O Parque é atravessado por rios de algumas das principais bacias hidrográficas do Brasil, tais como Taquarí (Bacia do rio Paraguai), Araguaia (Bacia do Rio Tocantins) e alguns tributários da Bacia do Rio Paranaíba, como os rios Sucuruí, Jacubá e Formoso (Benedito-Cecilio et al. 2004).

\section{Amostragem e identificação}

foram analisadas onze amostras de fauna associada a macrófitas (Tabela 1), obtidas entre abril de 2000 e novembro de 2006, sendo uma proveniente de vereda, duas da Lagoa das Capivaras e oito de campo úmido (seis próximas ao rio Formoso, uma da Cabeceira Alta e uma do córrego Buriti Torto).

Para a coleta das amostras foi utilizada rede de plâncton com abertura de malha de $80 \mu \mathrm{m}$, passada entre as macrófitas da zona

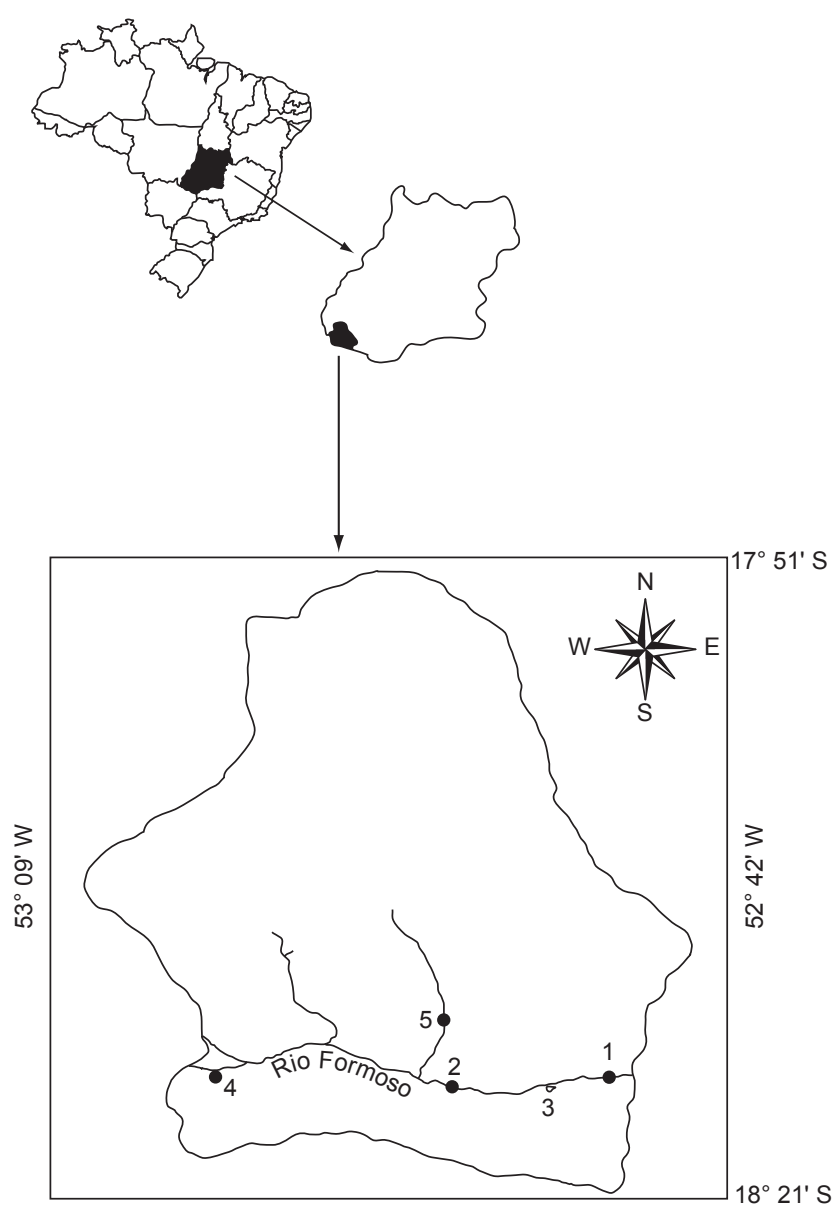

Figura 1. Localização do Parque Nacional das Emas-GO e dos pontos de amostragem: 1. Vereda; 2. Campo Úmido (Rio Formoso); 3. Lagoa das Capivaras; 4. Campo Úmido (Cabeceira alta - Rio Formoso); 5. Campo Úmido (Córrego Buriti Torto).

Figure 1. Localization of the Parque Nacional das Emas-GO and the sampling points: 1. Vereda; 2. Wet Campo (Formoso River); 3. Capivaras Lagoon; 4. Wet Campo (Cabeceira alta - Formoso River); 5. Wet Campo (Buriti Torto Stream).

marginal da vereda e da lagoa. No campo úmido, em que a profundidade da água impedia o arrasto da rede, esta era submersa entre as plantas, agitada e depois recolhida; este processo era repetido até que o reservatório coletor da rede estivesse completo. Para fixação das amostras, foi utilizado formol a $4 \%$.

As amostras foram triadas em microscópio estereoscópico em sua totalidade, quando possível; em caso de densidade elevada de animais, foram analisadas sub-amostras até que se obtivesse um número mínimo de 50 indivíduos; a partir desse ponto, novas sub-amostras eram triadas até que, em cinco consecutivas, o número de espécies não se alterasse. Os exemplares voucher encontram-se depositados no Laboratório de Zoologia da Universidade Católica de Brasília.

A identificação dos indivíduos foi feita em microscópio de contraste de fase (Carl Zeiss) e com auxílio de bibliografia especializada (Elmoor-Loureiro 1997, Dumont et al. 2002, Sinev 1998, 2001, Sinev \& Hollwedel 2002, Kotov \& Sinev 2004, Kotov \& Štifter 2006), considerando caracteres que são padrão para o grupo, tais como poros cefálicos, apêndices e pós-abdome.

As fotografias foram obtidas em microscópio óptico invertido através de câmera digital (Mitsuca ${ }^{\circledR}$ DC 7325BR), com 7.0 megapixels de resolução, diretamente da ocular do microscópio. 


\section{Resultados}

Foram encontradas 18 espécies tipicamente não planctônicas, distribuídas em três famílias: Ilyocryptidae, Macrothricidae e Chydoridae (Tabela 2; Figuras 2-4). A maior contribuição em número de espécies foi da família Chydoridae, com 15 táxons do total.

A Lagoa da Capivara e a vereda apresentaram maior número de espécies (12 e 11, respectivamente); destas, seis espécies ocorreram em ambos os ambientes (Tabela 2). Quatro espécies ocorreram exclusivamente nas amostras de vereda (Acroperus harpae, Alona iheringula, Alonella dadayi e Ephemeroporus tridentatus), enquanto que três foram exclusivas da Lagoa das Capivaras (Alona verrucosa, Graptoleberis occidentalis e Streblocerus pygmaeus).

Nos campos úmidos, foi observado menor número de espécies. No Buriti Torto e Cabeceira ocorreram seis espécies em cada ambiente, enquanto que no campo úmido do Rio Formoso foram encontradas apenas quatro espécies (Tabela 2). Nenhuma das espécies foi verificada exclusivamente em campo úmido.

Tabela 1. Amostras de fauna fitófila coletadas no Parque Nacional das Emas-GO.

Table 1. Samples of phytophilous fauna collected in the Parque Nacional das Emas-GO.

\begin{tabular}{|c|c|c|}
\hline Local de coleta & Coordenadas & Data \\
\hline Vereda & $18^{\circ} 16^{\prime} 10.9^{\prime \prime} \mathrm{S}$ e $52^{\circ} 45^{\prime} 17.9^{\prime \prime} \mathrm{W}$ & $21 / 04 / 00$ \\
\hline Lagoa das Capivaras & $18^{\circ} 16.245^{\prime} \mathrm{S}$ e $52^{\circ} 50.529^{\prime} \mathrm{W}$ & $09 / 12 / 00$ \\
\hline Lagoa das Capivaras & $18^{\circ} 16.244^{\prime} \mathrm{S}$ e $52^{\circ} 50.528^{\prime} \mathrm{W}$ & $09 / 12 / 00$ \\
\hline Campo úmido - R. Formoso & $18^{\circ} 15^{\prime} 42.9^{\prime \prime} \mathrm{S}$ e $52^{\circ} 53^{\prime} 23.2^{\prime \prime} \mathrm{W}$ & $22 / 04 / 00$ \\
\hline Campo úmido - R. Formoso & $18^{\circ} 15.567^{\prime} \mathrm{S}$ e $52^{\circ} 53.312^{\prime} \mathrm{W}$ & $07 / 12 / 00$ \\
\hline Campo úmido - R. Formoso & $18^{\circ} 15.733^{\prime} \mathrm{S}$ e $52^{\circ} 53.300^{\prime} \mathrm{W}$ & $07 / 12 / 00$ \\
\hline Campo úmido - R. Formoso & $18^{\circ} 15.567^{\prime} \mathrm{S}$ e $52^{\circ} 53.312^{\prime} \mathrm{W}$ & $06 / 02 / 01$ \\
\hline Campo úmido - R. Formoso & $18^{\circ} 15.567^{\prime} \mathrm{S}$ e $52^{\circ} 53.312^{\prime} \mathrm{W}$ & $22 / 04 / 01$ \\
\hline Campo úmido - R. Formoso & $18^{\circ} 15.567^{\prime} \mathrm{S}$ e $52^{\circ} 53.312^{\prime} \mathrm{W}$ & $01 / 06 / 01$ \\
\hline Campo úmido - Cabeceira Alta do R. Formoso & $18^{\circ} 15.591^{\prime} \mathrm{S}$ e $53^{\circ} 01.965^{\prime} \mathrm{W}$ & $13 / 11 / 06$ \\
\hline Campo úmido - Buriti torto & $18^{\circ} 14.276^{\prime} \mathrm{S}$ e $52^{\circ} 53.107^{\prime} \mathrm{W}$ & $14 / 11 / 06$ \\
\hline
\end{tabular}

Tabela 2. Ocorrência de espécies de cladóceros nas áreas amostradas no Parque Nacional de Emas-GO.

Table 2. Occurrence of cladocerans species in the sampling areas of the Parque Nacional de Emas-GO.

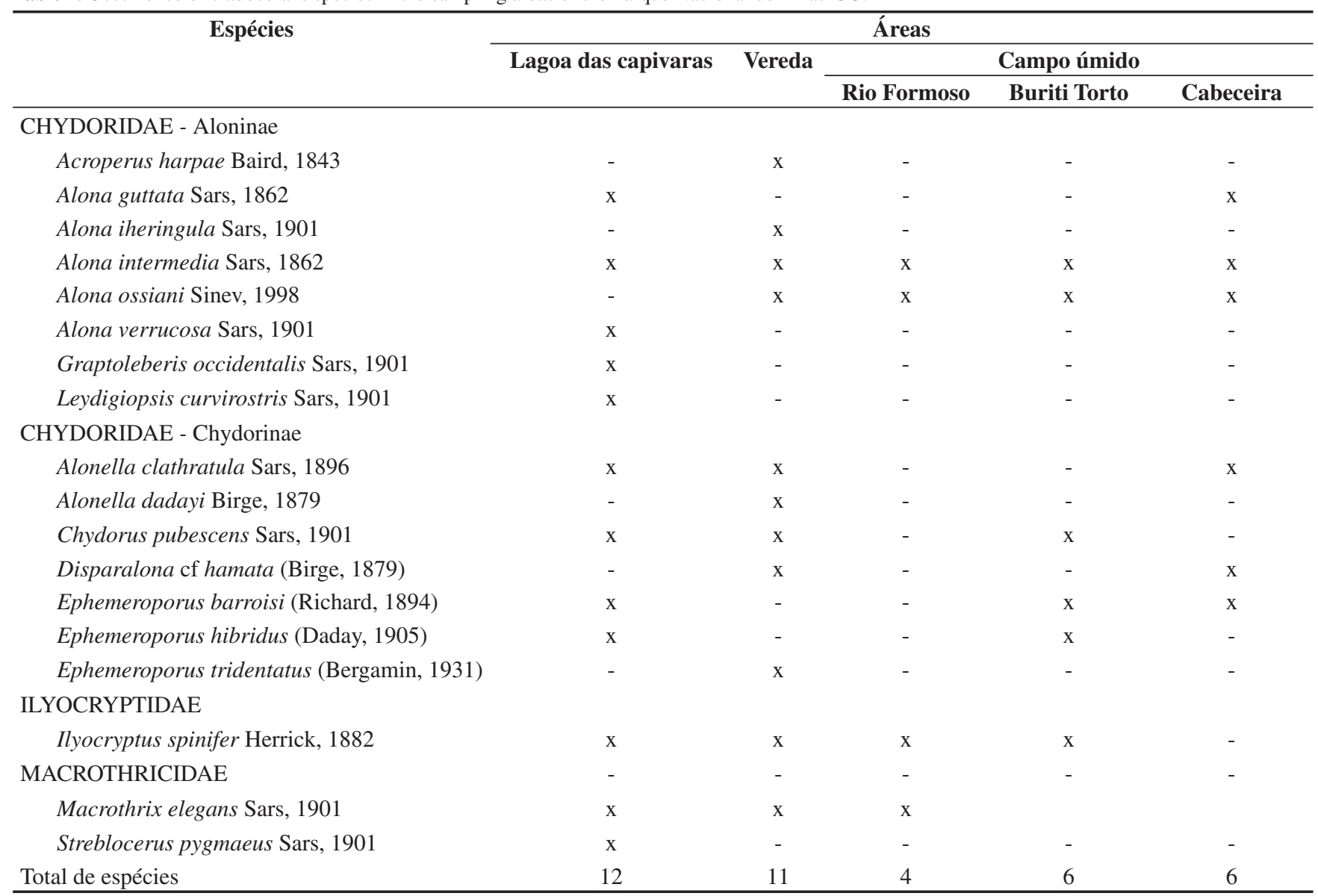



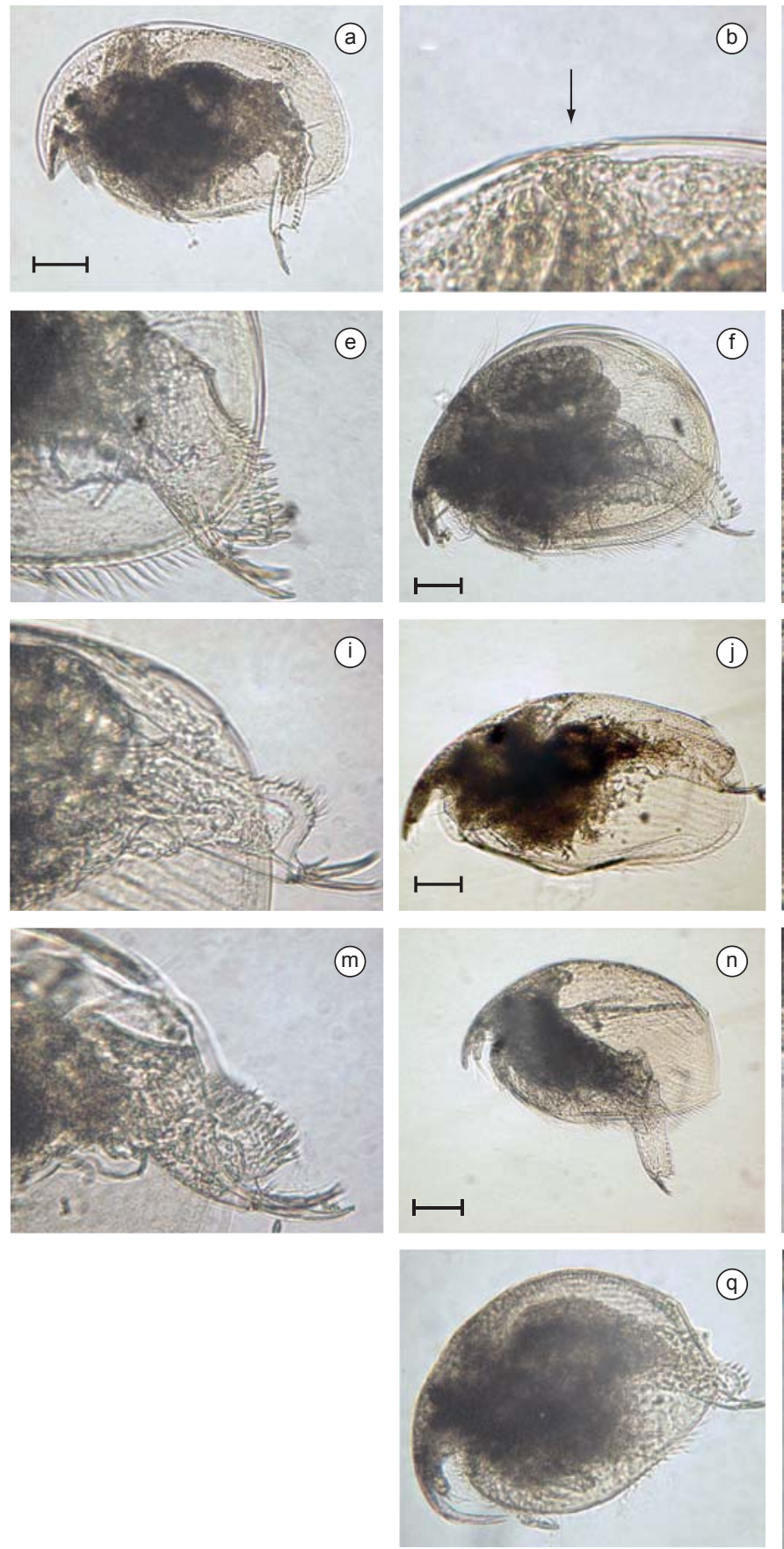

(b)
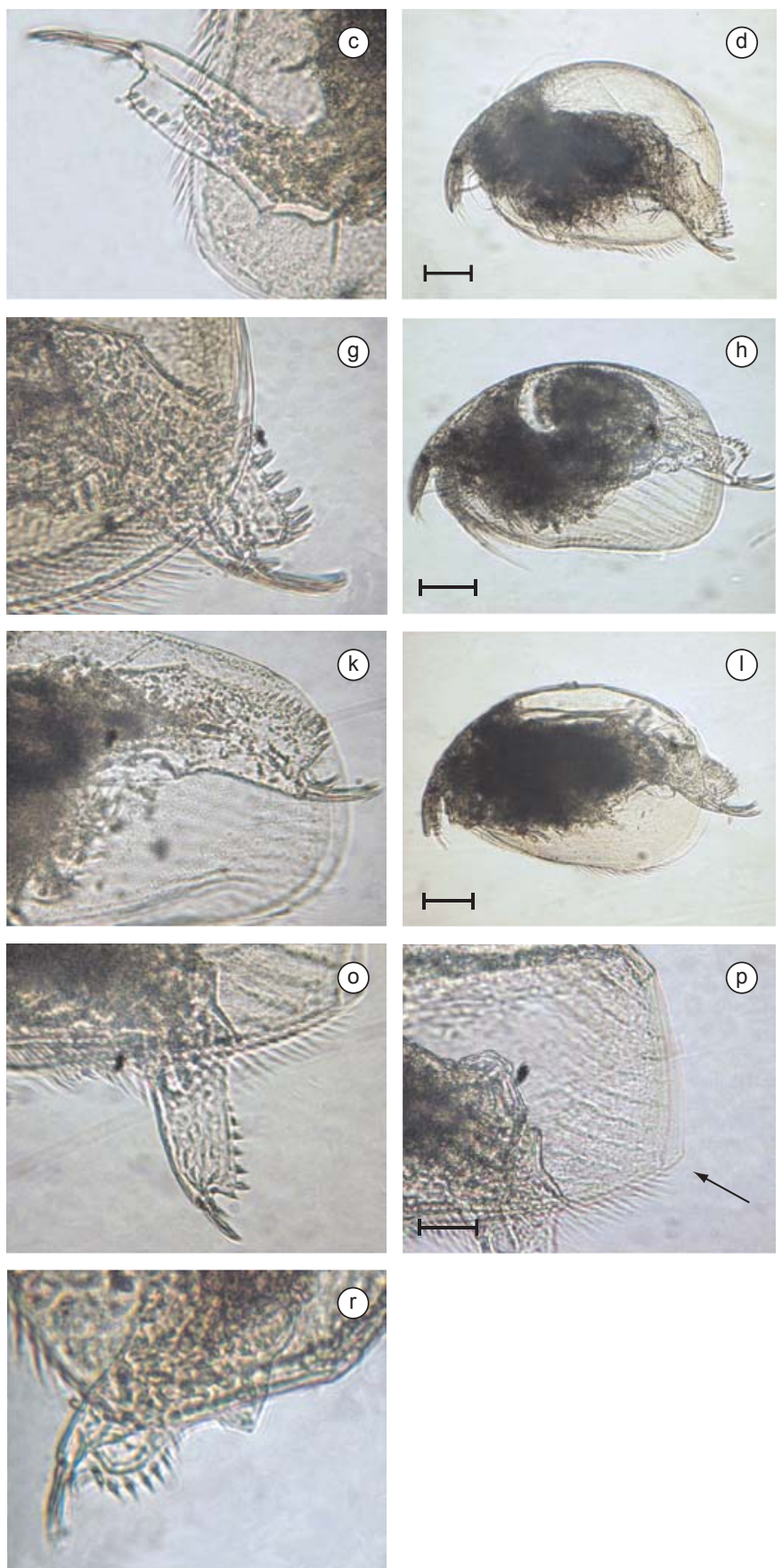

Figura 2. Cladóceros Chydoridae do Parque Nacional das Emas. a-c) Acroperus harpae: a) vista lateral; b) região dos poros cefálicos, indicados por seta; c) pós- abdome); d-e) Alona guttata: d) vista lateral; e) pós-abdome); f-g) Alona iheringula: f) vista lateral; g) pós-abdome; h-i) Alona intermedia: h) vista lateral; i) pós-abdome; j-k) Alona ossiani: j) vista lateral; k) pós-abdome; 1-m) Alona verrucosa: 1) vista lateral; m) pós-abdome; n-p) Alonella clathratula: n) vista lateral; o) pós-abdome; p) ângulo posterior ventral, indicado pela seta; q-r) Alonella daday: q) vista lateral; r) pós-abdome. Escalas = 100 $\mu \mathrm{m}$.

Figure 2. Chydoridae cladocerans from Parque Nacional das Emas. a-c) Acroperus harpae: a) lateral view; b) head pores, indicated by arrow; c) postabdomen; d-e) Alona guttata: d) lateral view; e) postabdomen; f-g) Alona iheringula: f) lateral view; g) postabdomen; h-i) Alona intermedia: h) lateral view; i) postabdomen; j-k). Alona ossiani: $\mathrm{j}$ ) lateral view; k) postabdomen; 1-m) Alona verrucosa: 1) lateral view; m) postabdomen; $\mathrm{n}-\mathrm{p}$ ) Alonella clathratula: $\mathrm{n}$ ) lateral view; o) postabdomen; p) postero-ventral angle, indicated by arrow; q-r) Alonella dadayi: q) lateral view; r) postabdomen. Scale bars = 100 um.

\section{Discussão}

O presente levantamento de espécies de Cladóceros, embora preliminar, é o primeiro para a região do Parque Nacional das Emas-GO. As espécies observadas têm ampla distribuição no Brasil, embora seja este o primeiro registro para o sudoeste de Goiás.
Este trabalho também pode ser considerado o primeiro com foco nos cladóceros de áreas alagadas (vereda e campo úmido) da região central do Brasil, embora Reid (1984), avaliando a meiofauna de campos úmidos no Distrito Federal, tenha informado a ocorrência de Alona iheringula (citada como Alona rustica). Esta espécie, no entanto, não foi registrada nos campos úmidos estudados, embora 


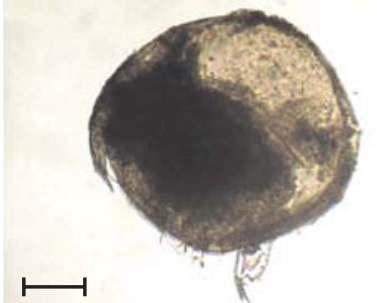

(a)
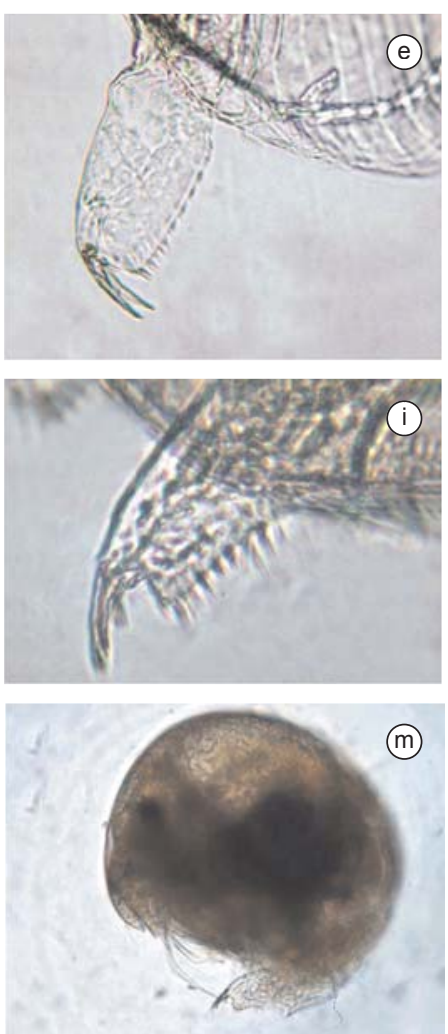

(m)
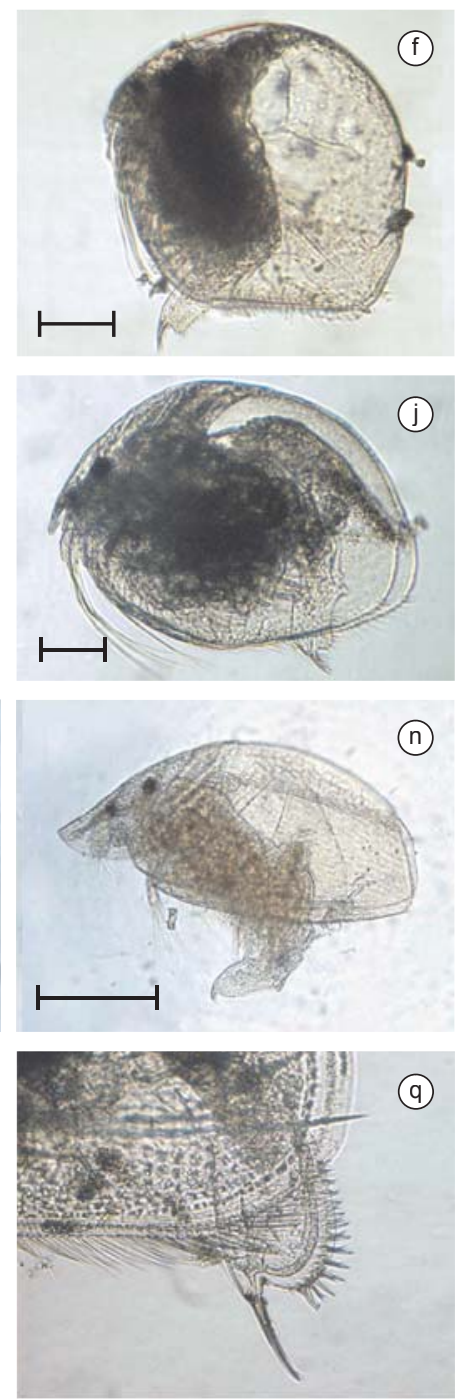
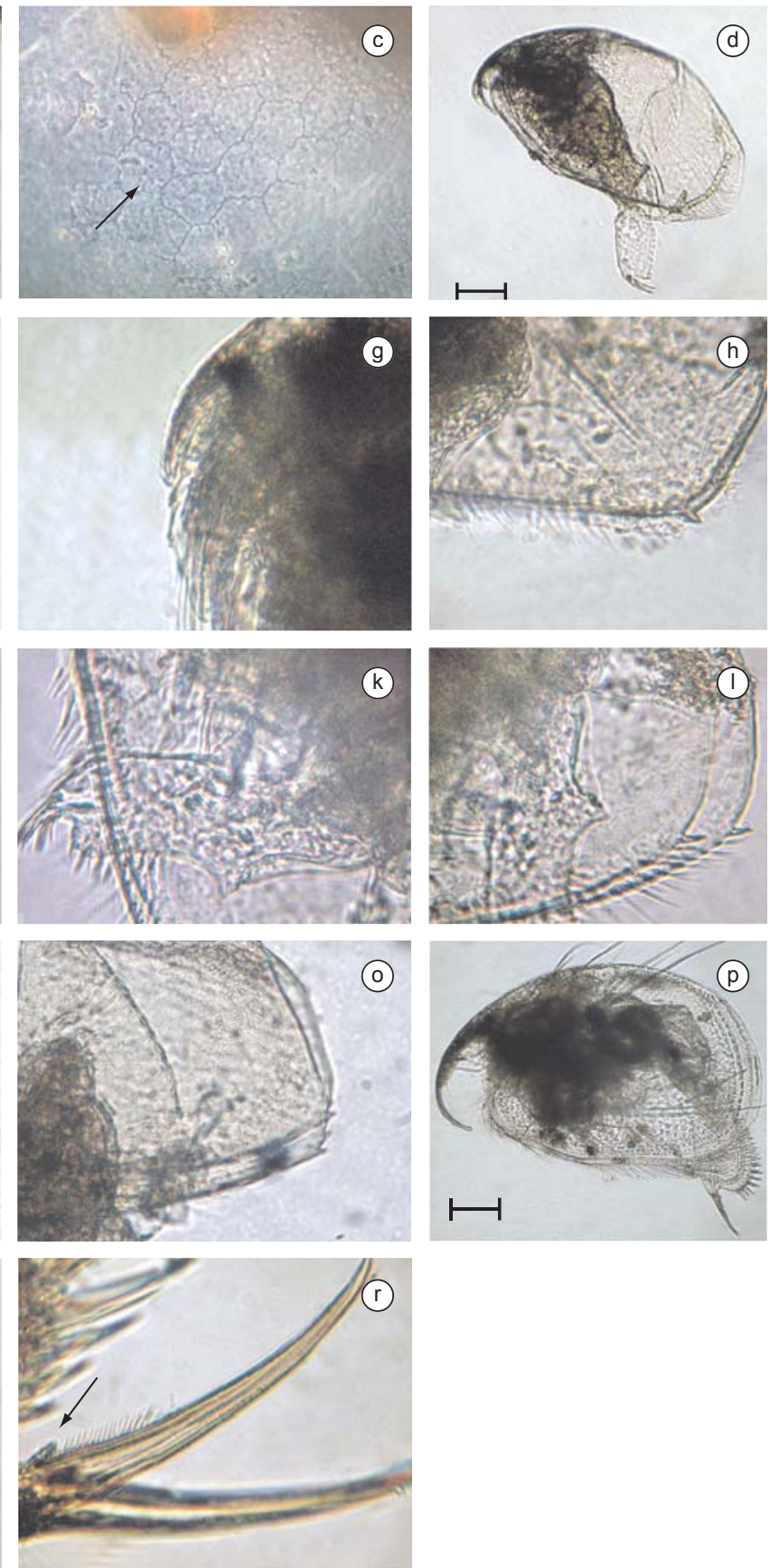

Figura 3. Cladóceros Chydoridae do Parque Nacional das Emas. a-c) Chydorus pubescens: a) vista lateral; b) pós-abdome; c) detalhe de um exemplar de Flores de Goiás, mostrando a superfície do escudo cefálico tipicamente marcada por linhas onduladas; d-e) Disparalona cf hamata: d) vista lateral; e) pós-abdome); f-i) Ephemeroporus barroisi s.l.: f) vista lateral; g) quilha do labro; h) ângulo posterior-ventral; i) pós-abdome); j-1) Ephemeroporus hybridus: j) vista lateral; k) pós-abdome; 1) ângulo posterior-ventral); m) Ephemeroporus tridentatus (vista lateral); n-o) Graptoleberis occidentalis: n) vista lateral; o) ângulo posteriorventral); p-r) Leydigiopsis curvirostris: p) vista lateral; q) pós-abdome; r) garra do pós-abdome, com seta indicando o espinho basal. Escalas $=100 \mu \mathrm{m}$.

Figure 3. Chydoridae cladocerans from Parque Nacional das Emas. a-c) Chydorus pubescens: a) lateral view; b) postabdomen; c) detail of a specimen from Flores de Goiás, showing headshield surface typically marked by wavy lines); d-e) Disparalona $\mathrm{cf}$ hamata: d) lateral view; e) postabdomen); f-i) Ephemeroporus barroisi s.l: f) lateral view; g) labral keel; h) postero-ventral angle; i) postabdomen); j-1) Ephemeroporus hybridus: j) lateral view; k) postabdomen; 1) posteroventral angle; m) Ephemeroporus tridentatus (lateral view); n-o) Graptoleberis occidentalis: n) lateral view; o) postero-ventral angle); p-r) Leydigiopsis curvirostris: p) lateral view; q) postabdomen; r) postabdominal claw, arrow indicates the basal spine. Scale bars $=100 \mu \mathrm{m}$.

estivesse presente nos ambientes de vereda e lagoa. É possível que a não ocorrência nas amostras de campo úmido do Parque Nacional de Emas seja devida à pequena profundidade da água nos pontos coletados, uma vez que Reid (1984) encontrou-a apenas em condições de maior profundidade de água.
A predominância de espécies de Chydoridae na fauna fitófila do Centro-Oeste brasileiro parece ser uma constante. Em estudo realizado na Lagoa Bonita na Estação Ecológica de Águas Emendadas-DF, foram encontradas 18 espécies de cladóceros fitófilos sendo, também a família Chydoridae a maior contribuinte em número de espécies, 

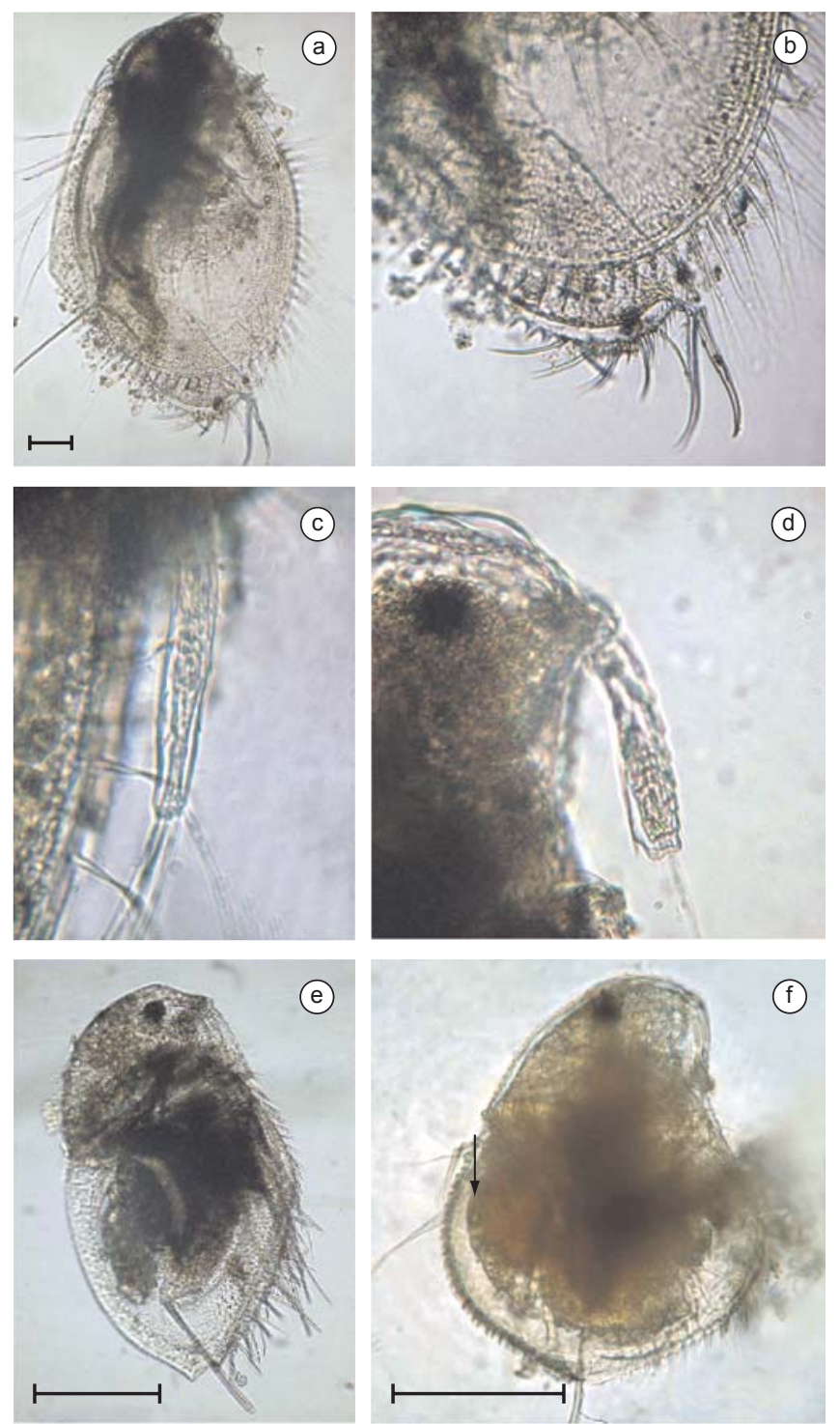

Figura 4. Cladóceros Ilyocrytidae e Macrothricidae do Parque Nacional da Emas. a-c) Ilyocryptus spinifer: a) vista lateral; b) pós-abdome; c) antênula; d-e) Macrothrix elegans: d) antênula; e) vista lateral;f) Streblocerus pygmaeus (vista lateral). Escalas $=100 \mu \mathrm{m}$.

Figure 4. Ilyocrytidae and Macrothricidae cladocerans from Parque Nacional das Emas. a-c) Ilyocryptus spinifer: a)lateral view; b) postabdomen; c) antennule); d-e) Macrothrix elegans: d) antennule; e) lateral view; f) Streblocerus pygmeaus (lateral view). Scale bars $=100 \mu \mathrm{m}$.

seguida dos Macrothricidae e Ilyocryptidae (Elmoor-Loureiro \& Mendonça-Galvão, no prelo). A maior riqueza de Chydoridae também foi verificada na fauna fitófila em corpos d'água diversos no vale do Rio Paranã, em Goiás (Elmoor-Loureiro 2007).

Todas as espécies encontradas são especializadas em explorar nichos proporcionados pelos bancos de macrófitas. A espécie Ilyocryptus spinifer (Figuras $4 \mathrm{a}-\mathrm{c}$ ) possui hábito bentônico, se associando com matéria orgânica floculada e podendo penetrar no sedimento (Kotov \& Štifter 2006). Sua ocorrência no campo úmido pode estar relacionada com a grande quantidade de sedimento rico em material orgânico proveniente da decomposição da vegetação, favorecendo sua colonização.

Os Macrothricidae (Figuras 4d-f) e os Chydoridae (Figuras 2-3) podem, igualmente, viver no fundo ou associados com a vegetação, possuindo apêndices especializados em raspar e conduzir o alimento e espinhos bem desenvolvidos, que também atuam na manipulação desses alimentos. Entre os Chydoridade, as especializações também incluem a maior espessura da carapaça e o uso efetivo do pós-abdome, que lhes permitiram maior mobilidade e sucesso neste tipo de ambiente (Fryer 1995).

No presente trabalho, verificou-se a ocorrência exclusiva de algumas espécies na vereda (A. harpae, A. iheringula, A. dadayi e E. tridentatus), na Lagoa das Capivaras (A. verrucosa, G. occidentalis, L. curvirostris e S. pygmaeus). Contudo, esses resultados são meramente sugestivos, considerando o caráter ocasional e assistemático da amostragem.

A menor riqueza nos ambientes de campo úmido (Tabela 2) pode estar relacionada à menor diversidade de nichos proporcionados pelas macrófitas. Além disso, visto que são sujeitos a um regime hídrico que alterna períodos de seca e submersão, os campos úmidos não favorecem o desenvolvimento do perifíton, condição para o estabelecimento de uma comunidade de cladóceros. As espécies observadas no campo úmido também estão presentes na vereda e/ou lagoa, sugerindo que a colonização das áreas úmidas ocorra a partir desses ambientes tipicamente lênticos.

Dentre as espécies registradas, oito merecem comentários sobre sua taxonomia. Inicialmente, cabe considerar a questão do gênero Biapertura, que vem sendo abandonado pelos especialistas, por concordarem que se trata de um táxon artificial, conforme sugerido por Frey (1987). Assim, as espécies atribuídas a este gênero por Smirnov (1971) têm sido transferidas para novos gêneros (Karualona, por exemplo) ou re-alocadas no gênero Alona, aguardando melhor avaliação de sua posição filogenética. Este é o caso de Alona intermedia Sars, 1862 (Figuras 2h-i) e Alona verrucosa Sars, 1901 (Figuras 2l-m); esta última já é assim tratada por Sinev \& Hollwedel (2002).

No final da década de 1980, difundiu-se o conceito do provável não cosmopolitismo das espécies de cladóceros (cf. Frey, 1987). Desde então, vários trabalhos têm dado suporte a essa idéia (dentre eles Sinev 1997, 1998, 2001a,b,c; Silva-Briano et al. 1999; Dumont \& Silva-Briano 2000; Dumont et al. 2002; Kotov et al. 2002), mostrando que espécies antes tidas como cosmopolitas representam complexos de espécies, semelhantes em suas características gerais, mas diversas quando se avança na observação de características mais finas, tais como seus apêndices torácicos.

A constatação de que Alona affinis (Leydig, 1860) representava um complexo de espécies, cada qual com distribuição regional, levou à descrição de Alona ossiani (Figuras 2j-k), membro do grupo com distribuição neotropical (Sinev 1998). Com base nas fortes evidências de não cosmopolitismo em cladóceros, anteriormente citadas, os autores do presente trabalho acreditam que os registros anteriores de $A$. affinis ou Biapertura affinis no Brasil possam ser atribuídos a A. ossiani. No caso particular do Parque Nacional de Emas, os exemplares concordam com a diagnose de A. ossiani (Sinev 1998). O mesmo tem sido observado em exemplares provenientes de outras regiões do Brasil (Elmoor-Loureiro, dados não publicados), o que dá suporte à idéia de que $A$. affinis stricto sensu não deva ocorrer no Brasil. Certamente, a re-avaliação taxonômica dos exemplares brasileiros atribuídos a $A$. affinis contribuiria para a resolução dessa questão.

Alona iheringula (Figuras $2 \mathrm{f}-\mathrm{g}$ ), sofreu um processo de atualização taxonômica em duas etapas. Sinev (2001a) reconheceu a validade da espécie sul-americana Alona iheringi Sars, 1901, considerada sinônimo júnior de Alona rustica Scott, 1895 (Smirnov 1971), recomendado a re-avaliação dos registros anteriores de A. rustica na América do Sul (cf. Elmoor-Loureiro 1997, para o Brasil). Mais tarde, a espécie foi renomeada como Alona iheringula, levando em consideração homonímia existente (Kotov \& Sinev 2004).

Alonella dadayi Birge, 1910 (Figuras 2q-r) tem ampla distribuição no Brasil (Elmoor-Loureiro 2000), também sendo citada como Disparalona dadayi (e.g. Elmoor-Loureiro 1997) e Phrixura dadayi 
(e.g., Campos et al. 1996, Lima et al. 2003). Smirnov (1996) afirma haver evidências para re-alocação dessa espécie em Alonella. Além disso, o nome Phrixura foi rejeitado pelo Comitê Internacional de Nomenclatura Zoológica (ICZN 2001). Assim, parece que Alonella dadayi seja o nome mais adequado para este táxon.

$\mathrm{O}$ crescente consenso sobre o não cosmopolitismo entre os Cladocera tem resultado na revisão e elevação de algumas subespécies ao status de espécie. Esta posição foi adotada por Paggi (1995) ao considerar Graptoleberis occidentalis Sars, 1901 (Figuras 3n-o) como espécie separada de Graptoleberis testudinaria (Fischer, 1851). A observação de detalhes finos das patas dos exemplares brasileiros (Elmoor-Loureiro, dados não publicados) corrobora a separação das duas espécies.

Até recentemente, a espécie Macrothrix elegans Sars, 1901 (Figuras 4d-e) constava como sinônimo júnior de Macrothrix triserialis Brady, 1886 (cf. Smirnov 1992). Em revisão do complexo Macrothrix rosea-triserialis, Dumont et al. (2002) revalidaram Macrothrix elegans. Kotov et al. (2004) confirmaram esta posição e mostraram a ampla distribuição dessa espécie no Neotrópico; esses autores também propõem que as referências sul-americanas para $M$. triserialis presentes em Smirnov (1992) e em Elmoor-Loureiro (1998) pertencem a M. elegans. De fato, a re-avaliação de exemplares do citado complexo, provenientes de diversas regiões do país, tem revelado tratar-se de M. elegans (Elmoor-Loureiro, dados não publicados), o que dá suporte à proposição da não ocorrência de $M$. triserialis no Brasil.

Cladóceros fitófilos podem ser utilizados como indicadores ecológicos, sendo necessário para isso compreender sua contribuição na manutenção e estabilidade de ecossistemas aquáticos, além de sólido conhecimento de sua distribuição e taxonomia (de Eyto et al. 2002). Essas condições, contudo, ainda não foram alcançadas no Brasil, sendo necessário ampliar a investigação científica sobre essa comunidade, nos seus mais diversos aspectos.

O presente trabalho cumpriu seu objetivo de apresentar o primeiro inventário, ainda que preliminar, dos cladóceros fitófilos presentes no Parque Nacional das Emas e região sudoeste de Goiás. Contudo, as 18 espécies observadas são de ampla distribuição no país, de modo que sua ocorrência na região não causa surpresa. Em comparação com a zona litoral da vereda e da lagoa, o campo úmido apresentou menor número de espécies, possivelmente em razão do menor desenvolvimento da comunidade perifítica em suas macrófitas.

\section{Agradecimentos}

Os autores são gratos aos colegas que disponibilizaram as amostras usadas no presente estudo; à Dra. Luciana de Mendonça Galvão pelo o incentivo, auxílio e, principalmente, amizade: a todos os integrantes do GEEA (Grupo de Estudos em Ecossistemas Aquáticos, UCB) pelo apoio e amizade; ao Programa de Iniciação Científica da Universidade Católica de Brasília, pela bolsa oferecida ao primeiro autor deste trabalho. Os autores agradecem a dois revisores anônimos pelas valiosas sugestões.

\section{Referências Bibliográficas}

BENEDITO-CECILIO, E., MINTE-VERA, C.V., ZAWADZKI, C.H., PAVANELLI, C.S., RODRIGUES, F.H.G. \& GIMENES, M.F. 2004. Ichthyofauna from the Emas National Park region: composition and structure. Braz. J. Biol. 64(3A):371-382.

CAMPOS, J.R.C., LANSAC-TÔHA, F.A., NUNES, M.A., GARCIA, A.P.P. \& PRADO, F.R. 1996. Composição da comunidade zooplanctônica de três lagoas da Ilha Porto Rico na planície de inundação do alto Rio Paraná. Acta Limnol. Bras. 8:183-194.

DE EYTO, E., IRVINE, K. \& FREE, G. 2002. The use of members of the family Chydoridae (Anamopoda, Branchiopoda) as an indicator of lake ecological quality in Ireland. Biol. Environ. 102B(2):81-91.
DUMONT, H.J. \& SILVA-BRIANO, M. 2000. Karualona n.gen. (Anomopoda: Chydoridae), with a description of two new species, and a key to all known species. Hydrobiologia 435:61-82.

DUMONT, H.J., SILVA-BRIANO, M. \& SUBHASH BABU, K.K. 2002. A re-evaluation of the Macrothrix rosea-triserialis group, with the description of two new species (Crustacea: Anomopoda: Macrothricidae). Hydrobiologia 467:1-44.

ELMOOR-LOUREIRO, L.M.A. 1997. Manual de identificação límnicos do Brasil. Editora Universa, Brasília.

ELMOOR-LOUREIRO, L.M.A. 1998. Branchiopoda. Freshwater Cladocera. In Catalogue of Crustacea of Brazil (P. S. Young, ed). Museu Nacional, Rio de Janeiro, p. 5-41.

ELMOOR-LOUREIRO, L.M.A. 2000. Brazilian cladoceran studies: where do we stand? Náuplius 8(1):117-131.

ELMOOR-LOUREIRO, L.M.A. 2007. Phytophilous cladocerans (Crustacea: Anomopoda and Ctenopoda) from Paranã River Valley, Goiás, Brazil. Rev. Bras. Zool. 24(2):344-352.

ELMOOR-LOUREIRO, L.M.A. \& MENDONÇA-GALVÃO, L. Lagoa Bonita: Fauna associada a macrófitas. In Reserva Ecológica de Águas Emendadas (SEMARH, org.) (no prelo).

FREY, D.G. 1987. The taxonomy and biogeography of the Cladocera. Hydrobiologia 145:5-17.

FRYER, G. 1995. Phylogeny and adaptive radiation within the Anomopoda: a preliminary exploration. Hydrobiologia 307:57-68.

INTERNATIONAL COMMISSION OF ZOOLOGICAL NOMENCLATURE. 2001. Opinion 1967-Disparalona Fryer, 1968 (Crustacea, Branchiopoda): conserved. Bull. Zool. Nomencl. 58(1):68-69.

KOTOV, A.A. \& SINEV, A.Y. 2004. Notes on Aloninae Dybowski \& Grochowski, 1894 emend. Frey, 1967 (Cladocera: Anomopoda: Chydoridae): 3. Alona iheringula nom. nov. instead of A. iheringi Sars, 1901, with comments on this taxon. Arthropoda Selecta 13(3):95-98.

KOTOV, A.A. \& ŠTIFTER, P. 2006. Cladocera: Family Ilyocryptidae (Branchiopoda: Cladocera: Anomopoda). Backhuys Publisher/Kenobi Productions, Leiden e Ghent.

KOTOV, A.A., DUMONT, H.J. \& VAN DAMME, K. 2002. Redescription of Ilyocryptus sarsi Stingelin, 1913. Hydrobiologia 472:207-222.

KOTOV, A.A., GARFIAS-ESPEJO, T. \& ELÍAS-GUTIÉRREZ, M. 2004. Separation of two Neotropical species: Macrothrix superaculeata (Smirnov, 1982) versus M. elegans Sars, 1901 (Macrothricidae, Anomopoda, Cladocera). Hydrobiologia 517:61-88.

LANSAC-TÔHA, F.A., VELHO, L.F.M. \& BONECKER, C.C. 1999. Estrutura da comunidade zooplanctônica antes e após a formação do reservatório de Corumbá-GO. In Ecologia de reservatórios: estrutura, função e aspectos sociais (R. Henry, ed). Fapesp/Fundibio, Botucatu, p.347-374.

LIMA, A.F., LANSAC-TÔHA, F.A., VELHO, L.F.M., BINI, L.M. \& TAKEDA, A.M. 2003. Composition and abundance of Cladocera (Crustacea) assemblages associated with Eichhornia azurea (Swartz) Kunth stands in the Upper Paraná River floodplain. Acta Sci., Biol. Sci. 25(1):41-48.

NOGUEIRA, M.G., GEORGE, D.G. \& JORCIN, A. 2003. Estudo do zooplâncton em zonas litorâneas lacustres: um enfoque metodológico. In Ecótonos nas interfaces dos Ecossistemas Aquáticos (R. Henry, ed). Editora Rima, São Carlos, p.83-127.

PADOVESI-FONSECA, C. 2005. Caracterização dos ecossistemas aquáticos do cerrado. In Cerrado: Ecologia, Biodiversidade e Conservação (A. Scariot, J.C. Sousa-Silva, \& J.M. Felfili, orgs). MMA, Brasília, DF, p.422-423.

PAGGI, J.C. 1995. Crustacea - Cladocera. In Ecosistema de Aguas Continentales: Metodologias para su estudio. (E.C. Lopretto \& G. Tell, eds). Ediciones Sur, La Plata, Tomo III, p.909-951.

RAIZER, J. \& AMARAL, M.E.C. 2001. Does the structural complexity of aquatic macrophytes explain the diversity of associated spider assemblages? J. Arachnol. 29:227-237.

REID, J.W. 1984. Semiterrestrial meiofauna inhabiting a wet campo in central Brazil, with special reference to the Copepoda (Crustacea). Hydrobiologia 118:95-111. 
REID, J.W. 1987. The cyclopoid copepods of a wet campo marsh in central Brazil. Hydrobiologia 153:121-138.

REID, J.W. 1993. The Harpacticoid and cyclopoid copepod fauna in the cerrado region of central Brazil. 1. Species composition, habitats, and zoogeography. Acta Limnol. Bras. 6:56-68.

REID, J.W. 1994. Murunducaris juneae, new genus, new species (Copepoda: Harpacticoida: Parastenocarididae) from a wet campo in central Brazil. J. Crustacean Biol. 14(4):771-781

SILVA-BRIANO, M., DIEU, N.Q. \& DUMONT, H.J. 1999. Redescription of Macrothrix laticornis (Jurine, 1820) and description of two new species of the M. laticornis-group. Hydrobiologia 403:39-61.

SINEV, A.Y. 1997. Review of the affinis-group of Alona Baird, 1843, with description of a new species from Australia (Anomopoda Chydoridae). Arthropoda Selecta 6(3/4):47-58.

SINEV, A.Y. 1998. Alona ossiani sp.n., a new species of the Alona affinis complex from Brazil, deriving from the collection of G.O. Sars (Anomopoda: Chydoridae). Arthropoda Selecta 7(2):103-110.

SINEV, A.Y. 2001a. Redescription of Alona iheringi Sars, 1901 (Chydoridae, Anomopoda, Branchiopoda), a South American species related to $A$. rustica Scott, 1895. Hydrobiologia 464:113-119.
SINEV, A.Y. 2001b. Separation of Alona cambouei Guerne \& Richard, 1893 from Alona pulchella King, 1853 (Branchiopoda: Anomopoda: Chydoridae). Arthropoda Selecta 10(1):5-18.

SINEV, A.Y. 2001c. Redescription of Alona glabra Sars, 1901, a South American species of the pulchella-group (Branchiopoda: Anomopoda: Chydoridae). Arthropoda Selecta 10(4):273-280.

SINEV, A.Y. \& HOLLWEDEL, W. 2002. Alona brandorffi sp.n. (Crustacea: Anomopoda: Chydoridae) - a new species from Brazil, related to $A$. verrucosa Sars, 1901. Hydrobiologia 472:131-140.

SMIRNOV, N.N. 1971. Chydoridae fauni Mira, Fauna of the USSR. Crustacea. $1(2): 1-531$.

SMIRNOV, N.N. 1992. The Macrothricidae of the world. SPB Academic Publishing, Amsterdam.

SMIRNOV, N.N. 1996. Cladocera: The Chydorinae and Sayciinae (Chydoridae) of the world. SPB Academic Publishing, Amsterdam.

Recebido em 20/05/07 Versão reformulada recebida em 02/11/07 Publicado em 07/02/08 